\title{
THE SHELL MODEL APPROACH-KEY TO HADRON STRUCTURE?
}

\section{HARRY J. LIPKIN}

Department of Nuclear Physics

Weizmann Institute of Science

Rehovot 76100 , Israel

and

School of Physics and Astronomy

=: Raymond and Beverly Sackler Faculty of Exact Sciences

Tel Aviv University

Tel Aviv, Israel

A shell model approach leads to a simple constituent quark model for hadron structure in which mesons and baryons consist only of constituent quarks. Hadron masses are the sums of the constituent quark effective masses and a hyperfine interaction inversely proportional to the product of these same masses. Hadron masses and magnetic moments are related by the assumption that the same effective mass parameter appears in the additive mass term, the hyperfine interaction, and the quark magnetic moment, both in mesons and baryons. The analysis pinpoints the physical assumptions needed for each relation and gives two new mass relations. Application to weak decays and recent polarized EMC data confirms conclusions previously obtained that the current quark contribution to the spin structure of the proton vanishes, but without need for the questionable assumption of SU(3) symmetry relating hyperon decays and proton structure. $\mathrm{SU}(3)$ symmetry breaking is clarified.

\section{THE EXTENDED AND UPDATED SAKHAROV-ZELDOVICH MODEL}

This paper presents an "Eastern view" of the shell-model approach to hadrons, in contrast to the "Western view" presented by Jaffe. Debates between these two views from regions separated by the line between time zone GMT +1 (Copenhagen) and GMT +2 (Rehovot) have long been traditional at Nuclear Physics Conferences. They continue analytically into the hadron domain.

The shell-model approach to hadrons began in 1966 with similar najve models proposed independently by two Eastern groups, Sakharov and Zeldovich in Moscow and Federman, Rubinstein and Talmi in Rehovot ${ }^{1,2}$ The model was rediscovered and refined with input from QCD by De Rujula, Georgi and Glashow ${ }^{3}$ in 1975 and further extended by Cohen and Lipkin ${ }^{4}$ in 1980. It begins with a standard general shell-model Hamiltonian, the sum of single-particle energies and a two-body interaction,

$$
H_{S M}=\sum_{i} \epsilon_{i}+\sum_{i>j} v_{i j}
$$

where $\epsilon_{i}$ is an effective single particle energy, $v_{i j}$ is a a two-body interaction and both $\epsilon_{i}$ and $v_{i j}$ are flavor dependent. One relation in excellent agreement with experiment immediately follows

-Work supported by the U.S. Department of Energy, Division of High Energy Physics, Contract W-31109-ENG-38. To be published in the Proceedings of the International Symposium on the Occasion of the 40th Anniversary of the Nuclear Shell Model, May 25-27, 1989. 
from the assumption that baryon mass splittings in the ground state configuration arise from the most general two-body forces with arbitrary spin and flavor dependence treated in first order using a single spatial "shell model wave function" with zero orbital angular momentum for all states in the configuration. ${ }^{2}$.

$$
M_{\Delta}-M_{N}=294 \mathrm{MeV}=2 M_{\Sigma}+M_{\Sigma}-3 M_{\Lambda}=307 \mathrm{MeV}
$$

The same assumptions also give the relation

$$
M_{\Xi *}-M_{\Xi}=216 \mathrm{MeV}=M_{\Sigma}-M_{\Sigma}=192 \mathrm{MeV}
$$

This $12 \%$ discrepancy is endemic in any model which uses two-body forces ${ }^{5}$ and is discussed below together with relations for the other states containing two or more strange quarks.

Sakharov and Zeldovich anticipated QCD by assuming that all the flavor dependence in the two-body interaction was in a flavor-dependent hyperfine interaction

$$
v_{i j}=v_{i j}^{o}+\vec{\sigma}_{i} \cdot \vec{\sigma}_{j} v_{i j}^{h y p}
$$

where $v_{i j}^{o}$ is independent of spin and flavor, $\vec{\sigma}_{i}$ is a quark spin operator and $v_{i j}^{\text {hyp }}$ is the strength of a flavor-dependent hyperfine interaction with different strengths but the same flavor dependence for quark-quark and quark-antiquark interactions. They obtained two relations between meson and baryon masses in surprising agreement with experiment ${ }^{1}$.

$$
\begin{gathered}
\epsilon_{s}-\epsilon_{u}=M_{\Lambda}-M_{N}=177 \mathrm{MeV}=\frac{3}{4}\left(M_{K^{*}}-M_{\rho}\right)+\frac{1}{4}\left(M_{K}-M_{\pi}\right)=180 \mathrm{MeV} \\
1.53=\frac{M_{\Delta}-M_{N}}{M_{\Sigma}-M_{\Sigma}}=\frac{M_{p}-M_{\pi}}{M_{K^{*}}-M_{K}}=1.61=\frac{v_{u d}^{\text {hyp }}}{v_{u s}^{\text {hyp }}}
\end{gathered}
$$

where the subscripts $u, d$ and $s$ refer to quark flavors. This striking evidence that mesons and baryons are made of the same quarks was overlooked for amusing reasons ${ }^{6,7}$ and rediscovered in $1978 .^{8}$

Input from QCD that the hyperfine interaction is produced by one gluon exchange explained the sign of the $\Delta-N$ and $\rho-\pi$ mass splittings ${ }^{3}$ and led to a successful prediction for $\mu_{\Lambda}$ obtained before the experiment ${ }^{3}$ by assuming that the ratio of the quark magnetic moments $\mu_{s}^{E M} / \mu_{d}^{E M}$ is the same as that of the corresponding color magnetic moments which produce the hyperfine splittings,

$$
\mu_{\Lambda}=-0.61 \mathrm{n} \cdot \mathrm{m} .=-\frac{\mu_{p}}{3} \frac{\mu_{s}^{E M}}{\mu_{d}^{E M}}=-\frac{\mu_{p}}{3} \frac{\mu_{s}^{c o l}}{\mu_{d}^{c o l}}=-\frac{\mu_{p}}{3} \frac{M_{\Sigma} \cdot-M_{\Sigma}}{M_{\Delta}-M_{N}}=-0.61 \mathrm{n} . \mathrm{m} .
$$

where $\mu_{f}^{\text {col }}$ denotes the color magnetic moment of a quark of flavor $f$. 
Further input from QCD suggests that the hyperfine interaction is inversely proportional to the product of the masses of the interacting quarks ${ }^{3}$ and otherwise flavor independent.

$$
v_{i j}^{h y p}=\frac{\vec{\sigma}_{i} \cdot \vec{\sigma}_{j}}{m_{i} m_{j}} \vec{v}_{i j}
$$

where $m_{j}$ is an effective quark mass and $\bar{v}_{i j}$ is flavor independent. The relation between this mass parameter $m_{i}$ and other quark mass parameters is not clear at this stage. However DGG introduced the assumption that the same mass parameters appear in the hyperfine interaction (1.6) and in the expression for the quark magnetic moments written as Dirac moments.

Cohen and Lipkin ${ }^{5}$ obtained a very simple and successful mass formula from the additional assumption that the same effective quark mass parameters $m_{i}$ appear also in the additive mass terms in the shell model Hamiltonian (1.1) and that these include not only the full single particle energy $\epsilon_{i}$ including the kinetic energy but also the spin and flavor independent part of the two-body interaction

$$
\begin{gathered}
\sum_{i} m_{i}=\sum_{i} \epsilon_{i}+\sum_{i>j} v_{i j}^{o} \\
M=\sum_{i} m_{i}+\sum_{i>j} \frac{\vec{\sigma}_{i} \cdot \vec{\sigma}_{j}}{m_{i} m_{j}} v_{i j} .
\end{gathered}
$$

Although the extreme assumption (1.7a) seems highly questionable the relation (1.7b) has described hadron masses and magnetic moments with remarkable success. This success remains to be understood at a more fundamental level. Some indications of the underlying physics has been given in one simple model ${ }^{4}$ which shows that the effective mass includes to a good approximation some relativistic corrections, kinetic energies and potential energies due to flavor and spin-independent effective quark-quark and quark-antiquark interactions related by the standard color factor of two., 90 These conclusions have been further supported by a variational treatment which shows that relations between baryon and meson masses like eqs. (1.4) and (1.8c) below are obtained as inequalities by using the exact three-body baryon wave function as a trial wave function for the meson case, and rescaling the wave function to satisfy the virial theorem. ${ }^{11}$

The original shell-model appoach was nonrelativistic, and the nonrelativistic model for constituent quarks described by an effective Lagrangian has been justified. ${ }^{12}$ However, the formula (1.7b) contains no explicit nonrelativistic dynamics and many relativistic effects have been shown $n^{4}$ to be included in the effective masses and effective interactions. The basic assumption that mesons and baryons consist of a constituent quark-antiquark pair and three constituent quarks respectively, with no additional constituents and no orbital angular momentum, determines the baryon wave function uniquely. The so-called $\mathrm{SU}(6)$ wave function is the only one that satisfies the requirements of Fermi statistics for colored quarks and is a color singlet with maximum space symmetry and zero orbital angular momeitum. 
The separation of orbital angular momentum can be questioned for the relativistic case, where a relativistic particle in a $j=1 / 2$ orbit with an s-wave large component has a $\mathrm{p}$-wave small component. Note, however, that a "relativistic shell model" can be defined in which each quark is described by a relativistic single-particle wave function with $j=1 / 2$ which is a solution of a Dirac equation, all three quarks in the baryon are in the same $j=1 / 2$ orbit in the unperturbed shell-model wave function, and the quark spin operators $\vec{\sigma}_{i}$ in eq. (1.7b) are replaced by the corresponding total quark angular momentum operators $\vec{j}_{i}$

Recently other models have been proposed with additional constituents ${ }^{13}$ motivated by recent experimental results in deep inelastic scattering and with different interactions ${ }^{14,15}$ motivated by an instanton picture. ${ }^{16}$ Such new models can be tested by comparing their predictions of masses and magnetic moments with those obtained from the simple model (1.7b). For this reason we examine the predictions of this model in detail, pinpointing the underlying assumptions for each successful prediction and discovering two new successful mass relations. We then use this model to examine the implications of the recent analyses of the polarized EMC result leading to the conclusion that the quark contribution to the proton spin is zero. ${ }^{17}$ We first obtain their result without their questionable assumption of $S U(3)$ relations between proton structure and hyperon decays and then examine the role of $S U(3)$ symmetry breaking.

To obtain nontrivial significant tests of the model we avoid taking credit for duplicating known mass formulas like Gell-Mann-Okubo which arise as good approximations in almost any model. Real tests of the model are obtained by considering only nine hadron masses not related by these simple formulas; those of the five baryons and four mesons in the ground state configuration which contain no more than one strange quark. Since the formula (1.7b) has four free parameters, the two quark masses and the two interaction strength parameters $v_{i j}$ for mesons and baryons, there are five independent relations. Two involve only baryons (five masses and three parameters), one involves only mesons (four masses and three parameters) and two relate mesons and baryons by assuming the equality of the two effective quark masses in both cases. We choose the the particular linear combinations of these relations which depend upon different underlying physical assumptions in simple ways. The remaining masses are then easily determined by using these nine as input into known mass formulas valid in nearly all models.

Two relations are (1.2a) and (1.4a). Assuming the hyperfine interaction inversely proportional to the same effective mass parameter $m_{i}$ in the first term of $(1.7 \mathrm{~b})$ gives two new relations, one with only baryons and one with only mesons,

$$
m_{s}-m_{u}=M_{\Lambda}-M_{N}=177 \mathrm{MeV}=\frac{M_{N}+M_{\Delta}}{6} \cdot\left(\frac{M_{\Delta}-M_{N}}{M_{\Sigma^{*}}-M_{\Sigma}}-1\right)=190 \mathrm{MeV}
$$


$m_{s}+m_{u}=\frac{3 M_{K^{*}}+M_{K}}{4}=793 \mathrm{MeV}=\frac{3 M_{\rho}+M_{\pi}}{8} \cdot\left(\frac{M_{\rho}-M_{\pi}}{M_{K^{*}}-M_{K}}+1\right)=791 \mathrm{MeV}$

Assuming the same effective mass parameter $m_{i}$ in the first spin-independent term in eq. (1.7b) for both mesons and baryons gives the relation

$$
m_{u}=\frac{M_{N}+\dot{M}_{\Delta}}{6}=362 \mathrm{MeV}=\frac{3}{8} \cdot M_{\rho}+\frac{1}{8} \cdot M_{\pi}=306 \mathrm{MeV}
$$

The relations for the quark mass difference (1.4a) and for the quark masses separately in baryons (1.8a) and mesons (1.8b) are in excelleat agreement with experiment, as is the relation between baryon hyperfine splittings (1.2a). Assuming the same quark mass in mesons and baryons gives a relation off by $13 \%$. The Sakharov-Zeldovich relation (1.4b) for the quark mass ratio is a linear combination of the poorer relation (1.2a) and the others and is therefore in between. The five reactions were choser to exhibit this basic physics, with four in excellent agreement and the $13 \%$ disagreement concentrated in a single relation. Assuming the same effective mass parameter in for both terms in eq.(1.7b) is thus an excellent approximation for baryons and mesons individually, and also for baryon magnetic moments (there are no data for meson magnetic moments). A $13 \%$ discrepancy from assuming the same effective masses in mesons and baryons appears to be additive for both flavors and does not appear in flavor differences.

The simplest explanation for this discrepancy, the change in potential energy due to the different sizes of the wave functions, is confirmed in the simple model mentioned abovet. Small corrections due to scaling the wave functions between mesons and baryons introduce a difference between the effective quark masses in mesons and baryons, thereby explaining the discrepancies in the relations (5) and (1.8c), with no appreciable effect on the quark mass difference.

We also note three predictions of hadron magnetic moments with no free parameters; namely (1.5) and

$$
\begin{gathered}
-1.46=\frac{\mu_{p}}{\mu_{n}}=-\frac{3}{2} \\
\mu_{p}+\mu_{n}=0.88 \mathrm{n} . \mathrm{m} .=\frac{2 M_{p}}{M_{N}+M_{\Delta}}=0.865 \mathrm{n} . \mathrm{m} .
\end{gathered}
$$

The well-known prediction for the ratio of the nucleon magnetic moments (1.9a) follows from the assumption that hadron magnetic moments are obtainable from the constituent quark wave functions with quark magnetic moments proportional to their electric charges. The relation (1.9b) was obtained by using Dirac moments for the quarks with effective quark masses determined from hadron masses and the first term of eq. (1.7b)..$^{18,19}$ The agreement with experiment of this prediction expressing a magnetic moment with a scale determined 
entirely by masses with no free parameters is impressive. Assuming the same parameter $m_{i}$ for both terms in eq. (1.7b) gave a new independent good prediction for $\mu_{\Lambda}{ }^{20}$

$$
-0.61=\mu_{\Lambda}=-\frac{M_{p}}{3 m_{s}}=\frac{-2 M_{p}}{M_{N}+M_{\Delta}+6\left(M_{\Lambda}-M_{p}\right)}=-\mathrm{C} .58
$$

This is now expressible in terms of eqs. (1.8a) and (1.8c) and its physical significance is better understood in terms of these relations.

That the simple model (1.7b) should succeed particularly well for masses of hadrons with no more than one strange quark can be seen by examining the perturbation series in a formulation beginning with an unperturbed zero-order Hamiltonian and wave function which are exact for a gedanken baryon with mass $M_{o}$ consisting of two nonstrange quarks and a third quark whose mass is $\left(m_{s}+m_{u}\right) / 2$. The exact mass of a nonstrange or singly strange baryon can then be expressed as a power series in the third quark mass difference $\left(m_{s}-m_{u}\right) / 2$,

$$
M_{B}=M_{0} \pm M_{1} \frac{\left(m_{s}-m_{u}\right)}{2}+M_{2} \frac{\left(m_{s}-m_{u}\right)^{2}}{4} \pm M_{3} \frac{\left(m_{s}-m_{u}\right)^{3}}{8}+\ldots \ldots
$$

where the plus sign is used for singly strange baryons and the minus sign for nonstrange. The coefficients $M_{n}$ in the perturbation series are determined by standard perturbation theory. The first coefficient $M_{1}$ is given by the Feynman-Hellmann theorem as a function of expectation value of derivatives with respect to the mass of the third quark of operators appearing in the mass operator (1.7b). Although we do not know the wave function of the gedanken baryon and cannot calculate the expectation values of the necessary operators, we can obtain meaningful results by leaving these expectation values as unknown parameters because the number of experimental masses known is greater than the number of parameters.

Our mass relations can thus be interpieted as first-order perturbation results calculated with a zero-order wave function corresponding to the exact wave function for the gedanken baryon. Our simple model therefore includes these first order contributions. Since the second order term contributes equally to the strange and nonstrange baryons and does not contribute to flavor mass differences, the first non-vanishing corrections to mass difference relations in our model are third order and express the error in using the same wave function for all baryons.

This treatment holds only for nonstrange and singly strange baryons, where the perturbation can be expressed as the mass change of only one quark in the hadron, and the expansion parameter is only half the flavor mass difference. A perturbation series valid for doubly strange hadrons will be a double series involving two quark mass differences, even when only $\Sigma$ and $\Xi$ masses are considered. A similar result is obtainable for mesons. Thus we see that the use of the same hadron wave function for the entire hadron $S U(3)$ multiplet and the neglect of differences in the wave function for different quark masses is a much better approximation 
for relations involving only nonstrange and singly strange hadrons than for relations involving masses of hadrons with more than one strange quark. Such a difference between the $\Sigma$ and $\Xi$ wave functions has been shown to explain the $12 \%$ disagreement in the relation $(1.2 b)^{5}$.

Predictions for the remaining ground state mesons and baryons containing two or three strange quarks and for the magnetic moments of the $\Sigma, \Xi$ and $\Omega$ hyperons are obtainable with no further free parameters but do not provide further significant tests of the model. The mass predictions are in reasonable agreement with experiment, but similar relations are obtained in limost any model; with small differences mainly due to the specific form of the strangeness dependence of the hyperfine interaction. Predictions of hyperon magnetic moments agree with experiment at the 15-20\% level, which may be good enough for such a crude model. However no attempts to do better have succeeded, since any attempts to introduce additional physical effects unavoidably introduce new parameters, and it is difficult to obtain a significant improvement in agreement with experiment.

It therefore seems reasonable to test any new proposed model which introduces different physics not contained in the model (1.7b) by its agreement with experiment in relating the masses of nonstrange and singly-strange mesons and baryons and its predictions of the nucleon and $\Lambda$ moments. Any disagreement with experiment in these cases is serious evidence that the physics which is left out of these models may be more important than the extra ingredient which has been put in.

\section{The New EMC Results and the Spin of the Proton.}

We now apply this constitient quark model to weak decays and the recent EMC experiment which has given rise to considerable controversy over the way in which quark spins contribute to the proton $\operatorname{spin}^{17}$. Tke standard analyses of the polarized EMC experiment to determine the spin structure of the proton ${ }^{17}$ introduce the quantities $\Delta u(p), \Delta d(p)$ and $\Delta s(p)$ which denote the fractional contributions of the $u, d$ and $s$ - flavored quarks and antiquarks respectively to the spin of the proton. The EMC result gives the relation

$$
\frac{4}{9} \Delta u(p)+\frac{1}{9} \Delta d(p)+\frac{1}{9} \Delta s(p)=0.246 \pm 0.026 \pm 0.056
$$

where the numbers on the right hand side come from the EMC data, multiplied by a factor of 1.08 from the perturbative $Q C D$ corrections introduced later on. ${ }^{17}$ This can be rewritten

$$
\Delta u(p)+\Delta d(p)+\Delta s(p)=1.11 \pm 0.12 \pm 0.25-[\Delta u(p)-\Delta d(p)]-\frac{1}{2}[\Delta d(p)-\Delta s(p)]
$$

The total contribution of the $u, d$ and $s$ - flavored quarks to the spin of the proton is thus expressed in terms of the EMC experimental result and two other quantities to be determined; namely $\Delta u(p)-\Delta d(p)$ and $\Delta d(p)-\Delta s(p)$. The $S U(6)$ wave function relates these two 
quantities and it is convenient to define a parameter $\xi$ which expresses the deviation of the experimental value of $\Delta d(p)-\Delta s(p)$ from the $S U(6)$ value.

$$
\begin{gathered}
{[\Delta d(p)-\Delta s(p)]_{S U(6)}=-\frac{1}{5}[\Delta u(p)-\Delta d(p)]_{S U(6)}} \\
\xi \equiv \frac{1}{2}[\Delta d(p)-\Delta s(p)]+\frac{1}{10}[\Delta u(p)-\Delta d(p)]
\end{gathered}
$$

BEK determine these quantities from weak decays. These decays, unlike the EMC experiment, do not measure directly a particular linear combination of $\Delta u(p), \Delta d(p)$ and $\Delta s(p)$. They measure flavor changing transition matrix elements which can be related to the spin contributions only via symmetry assumptions. The Wigner-Eckart theorem gives unique relations for transitions between states in the same multiplet of an $S U(2)$ subgroup of flavor $S U(3)$. These are most conveniently expressed as relations for $G_{A} / G_{V}$, since the vector and axialvector currents transform in the same way under $S U(3)$ and the Clebsch-Gordan coefficients arising in the application of the Wigner-Eckart theorem cancel out in the ratio.

Applying the Wigner-Eckart theorem to any $u \leftrightarrow d$ or any $u \leftrightarrow s$ transition between two states in the same isospin or V-spin multiplet gives

$$
\begin{aligned}
& G_{I}(u \leftrightarrow d)=\frac{\Delta u-\Delta d}{n_{u}-n_{d}} \\
& G_{V}(u \leftrightarrow s)=\frac{\Delta u-\Delta s}{n_{u}-n_{s}}
\end{aligned}
$$

where $\mathrm{G}(\mathrm{x})$ denotes the value of $g_{A} / g_{V}$ for the transition $\mathrm{x}, n_{f}$ denotes the number of quarks of flavor $f$ in the baryon under consideration, and the right hand side can be evaluated for any state in the isospin or V-spin multiplet. The relation (2.3a) which assumes only isospin symmetry is on firm ground. Relations like (2.3b) which assume nontrivial $S U(3)$ symmetry must be examined carefully to assess the effects of known $S U(3)$ symmetry breaking.

Applying (2.3a) to the neutron beta decay gives a quantity which we denote by

$$
\Delta_{u d}(N u c) \equiv \Delta u(p)-\Delta d(p)=\Delta d(n)-\Delta u(n)=G(n \rightarrow p)=1.259 \pm 0.004
$$

if isospin symmetry is assumed for the nucleon wave functions. Substituting Eqs. (2.2b) and (2.4) into eq. (2.1b) gives same result obtained in the original EMC analysis ${ }^{17}$ if the correction $\xi$ to the $\mathrm{SU}(6)$ value of $\Delta d(p)-\Delta s(p)$ is small; namely that the total quark spin contribution to the proton spin is very close to zero,

$$
\Delta u(p)+\Delta d(p)+\Delta s(p)=-0.02 \pm 0.12 \pm 0.25-\xi .
$$

We now use the constituent quark model to determine the value of $\xi$. For low momentum phenomenology, the structure of the constituent quark is not observed, and it is described with 
phenomenological parameters, including an effective constituent quark mass and an effective axial vector coupling. The observed value of $G_{A} / G_{V}$ for the nucleon is obtained ${ }^{21}$ by the trivial procedure of introducing one new free parameter to fit one new piece of data. From the $S U(6)$ wave function and setting

$$
\begin{gathered}
\left(\frac{g_{A}}{g_{V}}\right)_{\text {const }}^{\text {eff }}=\frac{3}{4}, \\
\left(\frac{G_{A}}{G_{V}}\right)_{\text {Nucleon }}=5 / 3\left(\frac{g_{A}}{g_{V}}\right)_{\text {const }}^{\text {eff }}=1.25
\end{gathered}
$$

which is close enough to the experimental value 1.259 for our purposes and allows us to work with simple fractions. This result has also been obtained with a chiral Lagrangian approach by Manohar and Georgi ${ }^{12}$.

We now apply this model to the EMC results and show that the experimental results are compatible with a model for the structure of the constituent quark in which each constituent quark contains a valence quark plus its share of the gluon and sea components of the hadron ${ }^{21}$. Deep inelastic scattering data are interpreted in terms of the current-quark partons for which $\left(g_{A} / g_{V}\right)=1$. Let $\delta u(f), \delta d(f)$ and $\delta s(f)$ denote the fractional contributions of the $u, d$ and $s$ - flavored current quarks and antiquarks respectively to the spin of a constituent quark of flavor $f$. We further decompose these fractional contributions into a valence contribution and a sea contribution, denoted by the superscripts $v$ and $s$ respectively.

The constituent quark of a given flavor has only a single valence quark of the same flavor and no contribution from valence quarks of a different flavor. Thus

$$
\delta u^{u}(d)=\delta d^{v}(u)=\delta s^{v}(d)=\delta s^{v}(u)=\mathbf{0}
$$

Applying eq. (2.3a) to the valence quark and substituting the value (2.6a) give

$$
\delta u^{v}(u)=\delta d^{v}(d)=3 / 4
$$

Since there are no valence strange quarks in the proton, and isospin but not $S U(3)$ symmetry is assumed for the sea quark contributions

$$
\begin{gathered}
\Delta s^{v}(p)=0 \\
\delta u^{s}(u)=\delta d^{s}(u)=\delta u^{s}(d)=\delta d^{s}(d) \\
\Delta u^{s}(p)=\Delta d^{s}(p) \\
\Delta u^{s}(p)=\Delta d^{s}(p)=(1+\epsilon) \Delta s^{s}(p)=(1+\epsilon) \Delta s
\end{gathered}
$$


where $\epsilon$ is an $S U(3)$-breaking parameter defining the excess of nonstrange quarks in the sea ${ }^{22}$. From (2.7b) and the SU(6) wave function expressed in terms of constituent quarks,

$$
\begin{gathered}
\Delta u^{v}(p)=-4 \delta d^{v}(p)=1 \\
\Delta u^{v}(p)+\Delta d^{v}(p)+\Delta s^{v}(p)=3 / 4
\end{gathered}
$$

If $\epsilon=0$, substituting eqs. (2.8a) and (2.9) into eq. (2.5) gives $\xi=0$. Thus the constituent quark model with the SU(6) wave function for the constituent quarks and an SU(3) symmetric sra again give the BEK result. The total quark spin contribution to the proton spin is very close to zero, but the valence quark contribution from $(2.10)$ is $3 / 4$ of the proton ipin. The sea contribution must canccl the quark contribution 22 and be $-(3 / 4) \times$ the proton pin. The proton spin therefore comes from elsewhere; e. g. gluons or orbital angular momentum. The peculiar result of $\mathrm{BEK}^{17}$ is now shifed to the constituent quark level, with a vanishing sum of valence and sea contributions to the constituent quark spin and with gluons and orbital angular momentum contributing the total spin of the constitnent quark. However, we have done more than simply shifting the problem elsewhere.

1. The same results obtained by ref. 17 have been obtained without use of any weak hyperon decay data and no assumptions of $S U(3)$ symmetry relating hyperon decays to proton structure.

2. The model used preserves all the good results of the constituent quark model, including the ratio $-(3 / 2)$ of the proton and neutron magnetic moments.

3. That the valence, sea and other contributions to the proton spin are all of order unity and conspire to give a total spin of $1 / 2$ for the proton is less mysterious at the quark level. The spin of a "dressed" elementary fermion is $1 / 2$ as the interactions which dress the fermion conserve angular momentum. The angular momentum of the lowest-lying composite bound state of three elementary fermions is not obvious.

\section{SU(3) Symmetry breaking and the Use of Hyperon Decay Data}

We now examine $\mathrm{SU}(3)$ symmetry breaking and the additional experimental input available from hyperon decays. We have used two of the three experimental inputs used by BEK ${ }^{17}$ to determine the three unknowns $\Delta u(p), \Delta d(p)$ and $\Delta s(p)$; namely the EMC result (2.1b) and the observed value of $G_{A} / G_{V}$ for the rucleon. Our third input eq. (2.9), comes from the standard constituent quark model wave function and can also be obtained by writing

$$
\frac{\mu_{p}}{\mu_{n}}=\frac{2 \Delta u^{v}(p)-\Delta d^{v}(p)}{2 \Delta d^{v}(p)-\Delta u^{v}(p)}=-\frac{3}{2} .
$$


We first consider SU(3) symmetry breaking when $\epsilon>0$; i.e. the sea contains a greater number of nonstrange than strange quark pairs. This has been discussed by Ramsey et al ${ }^{22}$ who introduced eq. (2.8d) and noted the cancellation by sea contributions of the valence quark contributions to the proton spin. We then obtain

$$
\xi=-\frac{3 \epsilon}{24+20 \epsilon}
$$

This correction to (2.5) is smaller than the experimental errors even with $\epsilon=1 / 2$.

The experimental data on semileptonic hyperon decays can be used as additional input or as a test of our model, with allowance for $\mathrm{SU}(3)$ symmetry breaking. We use the direct relations (2.2) between experimentally measured quantities and the proton spin contributions, rather than the conventional analysis with parameters like $D / F$ which have no direct physical interpretation. Applying eq. (2.3b) to the decay $\Sigma^{-} \rightarrow n+$ leptons gives a quantity which we denote by

$$
\begin{gathered}
\Delta_{u s}(H y p) \equiv \Delta u(n)-\Delta s(n)=\Delta d(p)-\Delta s(p)=\Delta s\left(\Sigma^{-}\right)-\Delta u\left(\Sigma^{-}\right)= \\
=G\left(\Sigma^{-} \rightarrow n\right)=-0.328 \pm 0.019
\end{gathered}
$$

by the assumption of SU(3) symmetry for the baryon wave functions. The experimental value ${ }^{23}$ disagrees by $4 \sigma$ with the SU(6) prediction (2.2a) using (2.4) and gives $\xi=-0.04 \pm 0.01$. However the correction to (2.5) is negligible.

Applying eq. (2.3b) to the decays $\Lambda \rightarrow p+$ leptons and $\Xi^{-} \rightarrow \Lambda+$ leptons is more complicated because the $\Lambda$ is not a $V$-spin eigenstate. Eq. (2.3b) gives

$$
G\left(V_{1} \rightarrow p\right)=G\left(\Xi^{-} \rightarrow V_{1}\right)=(1 / 2) \Delta_{u s}(H y p),
$$

where $V_{1}$ denotes the linear combination of $\Lambda$ and $\Sigma^{\circ}$ which is a member of a $V$-spin vector, and whose projections on the physical states are given by

$$
\left|\left\langle\Lambda \mid V_{1}\right\rangle\right|^{2}=3\left|\left\langle\Sigma^{\circ} \mid V_{1}\right\rangle\right|^{2}=3 / 4
$$

We also note the $S U(3)$ relations,

$$
\begin{aligned}
& \Delta u(p)=\Delta d(n)=\Delta u\left(\Sigma^{+}\right)=\Delta d\left(\Sigma^{-}\right)=\Delta s\left(\Xi^{-}\right)=\Delta s\left(\Xi^{o}\right) \\
& \Delta d(p)=\Delta u(n)=\Delta s\left(\Sigma^{+}\right)=\Delta s\left(\Sigma^{-}\right)=\Delta d\left(\Xi^{-}\right)=\Delta u\left(\Xi^{o}\right) \\
& \Delta s(p)=\Delta s(n)=\Delta d\left(\Sigma^{+}\right)=\Delta u\left(\Sigma^{-}\right)=\Delta u\left(\Xi^{-}\right)=\Delta d\left(\Xi^{o}\right)
\end{aligned}
$$

From eqs. (3.4) and SU(3) relations we obtain

$$
G\left(\Xi^{-} \rightarrow \Lambda\right)=(1 / 3)[\Delta u(p)+\Delta d(p)-2 \Delta s(p)]=(1 / 3) G(n \rightarrow p)+(2 / 3) G\left(\Sigma^{-} \rightarrow n\right)
$$


$G(\Lambda \rightarrow p)=(1 / 3)[2 \Delta u(p)-\Delta d(p)-\Delta s(p)]=(2 / 3) G(n \rightarrow p)+(1 / 3) G\left(\Sigma^{-} \rightarrow n\right)$

Substituting eq. (3.3) and the experimental values ${ }^{24}$ into eqs. (3.6) gives

$$
\begin{gathered}
\Delta_{u s}(H y p)=G\left(\Sigma^{-} \rightarrow n\right)=(3 / 2) G\left(\Xi^{-} \rightarrow \Lambda\right)-(1 / 2) G(n \rightarrow p)=-0.25 \pm 0.08 \\
\Delta_{u s}(H y p)=G\left(\Sigma^{-} \rightarrow n\right)=3 G(\Lambda \rightarrow p)-2 G(n \rightarrow p)=-0.43 \pm 0.07
\end{gathered}
$$

Eqs. (3.3) and (3.7) show that the hyperon beta decays all give the same parameter $\Delta_{u s}($ Hyp $)$ with the more accurately measured $\Delta_{u d}(N u c)$ as input and that the SU(3) prediction of equality is consistent with experiment at the $2 \sigma$ level. The magnitude of the discrepancy is expressed by the values of our correction term (2.2b), which is zero in our model and should have a definite value if $\mathrm{SU}(3)$ symmetry holds. The experimental values (3.3) and (3.7) place $\xi$ in the range

$$
-0.09 \pm 0.04 \leq \xi \equiv(1 / 2)\left[0.25+\Delta_{u s}(H y p)\right] \leq 0 \pm 0.04
$$

This range is smaller than the other errors in the expression (2.5) and includes the value $\xi=0$ of the simple model. Thus our result is essentially the same as that obtained ${ }^{17}$ by using the hyperon data as input. The errors introduced by using SU(3) relations may be appreciable; however they are still down in the noise when using the present EMC data to determine the contribution of the quarks to the spin of the proton.

Two additional $S U(3)$-breaking effects are flavor asymmetry in the sea and configuration mixing in the valence quarks. In any model where baryons contain a sea of quark-antiquark pairs which is not flavor symmetric ${ }^{22}$ the second equality in eq. (3.3) is clearly violated. It holds for the valence quarks, but implies that the sea in the $\Sigma^{-}$contains less $u$ quarks than $s$ quarks and is seen to be inconsistent with the relations (3.5) and isospin. Breaking SU(3) in the nucleon wave function means breaking isospin in the $\Sigma^{-}$wave function if the $S U(3)$ relations (3.5) are used. Thus the treatment used to determine $\Delta u(p), \Delta d(p)$ and $\Delta s(p)$ with the aid of $S U(3)$ symmetry and hyperon data is incorrect for a model with a sea which breaks $S U(3)$ syr:metry and which is essentially the same in nucleons and hyperons.

This effect might be treated by assuming that the hyperon decays involve only the valence quarks with a spectator sea, since a flavor-changing operator on the sea will not give a flavorneutral sea and will have a negligible overlap with the final baryon wave function. In this case eq. (2.3b) can be used for the valence quarks alone. This assumption can be justified in a model which assumes an $S U(3)$ classification only for the weak currents and not for the hadron spectrum. The hyperon decay data therefore give the quantity

$$
G\left(\Sigma^{-} \rightarrow n\right)=\Delta_{u s}^{v}(H y p) \equiv \Delta^{v} u(n)-\Delta^{v} s(n)=\Delta^{v} s\left(\Sigma^{-}\right)-\Delta^{v} u\left(\Sigma^{-}\right)
$$

where the superscript denotes that only valence contributions are considered. This effect can now be taken into account and leads to the same additional small correction term appearing 
in eq. (3.2). Thus we can obtain an expression valid for the case where the sea is not $S U(3)$ symmetric. The correction term $\xi$ is now given by

$$
\xi=(1 / 2)\left[0.25+\Delta_{u s}(H y p)\right]-\frac{3 \epsilon}{24+20 \epsilon}
$$

This is one example where the simple $S U(3)$ assumption is clearly invalid, but an understanding of the way in which $S U(3)$ is broken still leads to useful relations.

Further $S U(3)$ breaking arises with configuration mixing, implied if the hyperon decay data do not give the $S U(6)$ value $\Delta_{u s}(H y p)=-0.25$. All models for configuration mixing also introduce strong $S U(3)$ breaking, primarily because the dominant mechanism for $S U(6)$ breaking, the two-body tensor force, depends upon quark masses. The simplest example, a $D$-wave admixture analogous to the case of the deuteron in nuclear physics, admixes an $S U(3)$ decuplet with orbital angular momentum $\mathrm{L}=2$ and quark spin $\mathrm{S}=3 / 2$. Such octet-decuplet mixing can occur in the $\Sigma$ and $\Xi$, but is isospin forbidden in the nucleon and $\Lambda$. This mixing cannot be described by a " $D / F$ ratio" and any attempt to use hyperon data in this formalism to obtain information about nucleon spin structure is highly questionable. In fact such mixing can explain the $S U(6)$ violations in hyperon data without affecting the spin structure of the aucleon and retaining the relation $(2.2 \mathrm{a})$.

We therefore conclude that the simple constituent quark model gives a remarkably good description of low energy hadron spectroscopy and also of weak decays and the spin structure of the proton. However, the structure of the constituent quarks and the values of their effective masses and couplings remains a mystery to be resolved by QCD. The questions raised by the evidence for peculiar spin structure in the proton are shifted to the quark level, where it appears as peculiar spin structure for the constituent quark.

\section{REFERENCES}

1. Ya. B. Zeldovich and A.D. Sakharov, Yad. Fiz 4 (1966) 395; Sov. J. Nucl. Phys. 4 (1967) 283.

2. P. Federman, H.R. Rubinstein and I. Talmi, Phys. Lett. 22 (1966) 203; H.R. Rubinstein, Phys. Lett. 22 (1966) 210.

3. A. De Rujula, H. Georgi and S.L. Glashow, Phys. Rev. D12 (1975) 147.

4. I. Cohen and H. J. Lipkin, Phys. Lett. 93B, (1980) 56.

5. I. Cohen and H. J. Lipkin, Phys. Lett. 106B, (19S1) 119.

6. Harry J. Lipkin, in Gauge Theories, Massive Neutrinos and Proton Decay (Proceedings of Orbis Scientiae 18th Annual Meeting, Coral Gables, Florida, 1981) edited by Behram Kursonoglu and Arnold Perlmutter (Plenum, N.Y., 19S1), p. 359. 
7. A. D. Sakharov, private communication; Harry J. Lipkin, Annals of the New York Academy of Sciences 452 (1985) 79, and London Times Higher Education Supplement, January 20 (1984), p.17.

8. Harry J. Lipkin, Phys. Lett. B74 (1978) 399.

9. H.J. Lipkin, Phys. Lett. 45B (1973) 267.

10. H. J. Lipkin, Phys. Lett. 171B, 293 (1986).

11. H. J. Lipkin, Phys. Lett. 172 (1986) 242.

12. Aneesh Manohar and Howard Georgi, Nucl. Phys. B234, 189 (1984).

13. John Ellis, Erwin Gabathuler and Marek Karliner, Phys. Lett. B217 (1989) 173.

14. A. Arima and K. Yazaki, Phys. Lett. B183 (1987) 131.

15. E. V. Shuryak and Jonathan L. Rosner, Phys. Lett. B218 (1989) 72.

16. D. Horn and S. Yankielowicz, Phys. Lett. B76 (1978) 343.

17. Stanley J. Brodsky, John Ellis and Marek Karliner, Phys. Lett. 206B, 309 (1988).

18. Harry J. Lipkin, In: Proceedings of the 7th International Symposium on High Energy Spin Physics, Protvino (USSR), 22-27 September, (1986) Edited and Published by The Institute of High Energy Physics, Serpukhov (USSR), (1987) Vol, I, p.98.

19. Harry J. Lipkin, Nucl. Phys. A478, (1988) 307c.

20. H.J. Lipkin, Phys. Rev. Lett. 41 (1978) 1629/

21. Harry J. Lipkin, Nucl. Phys. A446, (1985) 409c.

22. G. Ramsey et all Phys. Rev. D39, 361 (1989).

23. S. Y. Hsueh et al, Phys. Rev. D38 (1988) 2056.

24. Particle Data Group, Phys. Lett. B204 (1988) 1.

\title{
DISCLAIMER
}

\begin{abstract}
This report was prepared as an account of work sponsored by an agency of the United States Government. Neither the United States Government nor any agency thereof, nor any of their employees, makes any warranty. express or inplied, or assumes any legal liability or responsibility for the accuracy, completeness, or usefulness of any information, apparatus, product, or process disclosed, or represents that its use would not infringe privately owned rights. Reference herein to any specific commercial product, process, or service by trade name, trademark, manufacturer, or otherwise does not necessarily constitute or imply its endorsement, recomnendation, or favoring by the United States Government or any agency thereor. The views and opinions of authors expressed herein do not necessarily state or reflect those of the United States Government or any agency thereof,
\end{abstract}

